\title{
Retraction of: DNA Cell Biol, 2017; 36 [Epub ahead of print]; DOI: $10.1089 /$ dna.2017.3798
}

DNA and Cell Biology $(D N A \& C B)$ is officially retracting the paper by Hongjing Zhao, Yu Wang, Juanjuan Liu, Yizhi Shao, Jinglun Li, Hongliang Chai, Mingwei Xing, entitled, "Molecular Characterization and Biological activity of Interferon- $\alpha$ in Indian Peafowl (Pavo cristatus)" (DNA Cell Biol, 2017; 36 [Epub ahead of print]; DOI: 10.1089/dna.2017.3798).

The Editor-in-Chief of $D N A \& C B$, Dr. Carol Shoshkes Reiss, was alerted to a discrepancy between the findings in the article by Zhao et al. and those of others, about the absence of expression of ISG15 in chickens. Dr. Reiss requested from the authors a clarification in their observations and inquired about the failure to include relevant citations in the reference section of the paper.

Based on the response from the authors, it appeared that they did not have the confidence in the data as they were not able to repeat the experiments, and were also unsure of the molecular probes that were used in the study. Therefore, the Editor has determined that the paper should be officially retracted from DNA and Cell Biology. 